\title{
Reaction of tungsten anion clusters with molecular and atomic nitrogen
}

\author{
Young Dok Kim, ${ }^{\text {a) }}$ Davor Stolcic, Matthias Fischer, and Gerd Ganteför \\ Fachbereich Physik, Universität Konstanz, D-78457 Konstanz, Germany
}

\begin{abstract}
Ultraviolet photoelectron spectra for $\mathrm{W}_{n} \mathrm{~N}_{2}^{-}(n=1-8)$ clusters produced by addition of atomic and molecular nitrogen on $\mathrm{W}$ anion clusters are presented. Evidence is provided that molecular chemisorption of $\mathrm{N}_{2}$ is more stable than the dissociative one on tungsten anion clusters consisting of eight atoms or less, which is completely different from the results on tungsten bulk surfaces. A general tendency toward molecular chemisorption for small clusters can be explained by reduced charge transfer from the metallic $d$-orbitals of cluster in contrast to bulk $d$-orbitals. Comparative studies on chemisorption of atomic and molecular species of diatomic molecules on nanoclusters are expected to provide important information on size-dependent changes of chemical properties of nanoclusters.
\end{abstract}

\section{INTRODUCTION}

Adsorptive behaviors of gas molecules on metal clusters change significantly as a function of cluster size, leading to the interesting cluster size effects on catalytic reactivities. ${ }^{1-16}$ For example, oxygen adsorption reactivities of $\mathrm{Au}$ anion clusters exhibit an even/odd alternation, i.e., Au anion clusters with even numbered gold atoms are active for oxygen adsorption, whereas odd-numbered clusters are inert. ${ }^{5}$ Rates of chemisorption for $\mathrm{H}_{2}, \mathrm{D}_{2}$, and $\mathrm{N}_{2}$ on $\mathrm{Fe}_{n}$ (Refs. 8 and 9) and $\mathrm{Nb}_{n}$ clusters, ${ }^{10-14}$ and $\mathrm{CO}$ on different metal clusters ${ }^{15}$ vary by several orders of magnitudes as a function of $n$. Tungsten clusters ( $\mathrm{W}_{n}, n=$ number of atoms) with $n<15$ show relatively low reactivities toward nitrogen adsorption, whereas a sharp jump of the reactivity is detected for $n$ $=15$ at liquid-nitrogen temperature as well as room temperature. ${ }^{6,7}$ In many cases, correlations between electronic structures (for example, ionization potentials) and chemisorption reactivities of metal clusters have been found. $1,2,5,8-10,13,14,16$

Chemisorption experiments can end up with a metastable state, when further transitions to other metastable states or to the ground state are kinetically hindered, thus determination of the ground state for the chemisorption based on experimental observations can be misleading. For example, dissociation of nitrogen molecules on metal surfaces, which is the rate-determining step of the ammonia synthesis, can involve large activation barriers, and therefore, only molecular chemisorption can be observed in the ultrahigh vacuum conditions, even though dissociation is thermodynamically more favored with respect to the molecular chemisorption. ${ }^{17}$ Under conditions of real catalysis (higher pressures, higher temperatures), high activation barriers can be easily overcome, and consequently chemical species, which are not identified in scientific experiments, can form on the surface, opening unexpected reaction channels. To acquire a molecular-level understanding for the mechanism of real world chemistry, information on complete energetics

${ }^{a)}$ Electronic mail: young.kim@uni-konstanz.de of chemisorption is required, which can be obtained by developing new experimental techniques to facilitate the kinetically hindered reactions steps.

In this paper, interactions of tungsten anion clusters with atomic nitrogen and molecular nitrogen were compared using time of flight (TOF) mass spectroscopy and ultraviolet photoelectron spectroscopy (UPS). Dissociation of nitrogen was found to be less stable with respect to the molecular chemisorption on $\mathrm{W}_{n}^{-}$clusters for $n=1-8$, which is completely different from the results for tungsten bulk crystals. ${ }^{18,19}$ This result can be explained by the different properties of the metallic $d$-orbitals with lower coordination numbers in small clusters: The less effective screening caused by the smaller number of directly neighboring atoms hampers charge transfer from the metal to the antibonding $\pi^{*}$ orbital of the adsorbate. Consequently, the adsorbate is not dissociated, but molecularly bound with the intermolecular bonding being highly activated. Our result suggests that strong cluster-size-dependent changes of catalytic activities may be present for the reactions which involve nitrogen adsorption.

\section{EXPERIMENTAL DETAILS}

Our experimental setup has been described in detail elsewhere. ${ }^{20}$ Briefly, tungsten clusters are produced with a pulsed arc cluster ion source in which tungsten is evaporated by a pulsed electric arc within a carrier gas $(\mathrm{He})$ injected using a pulsed valve. He gas pulse flushes the evaporated material through a $10-\mathrm{cm}$-long channel (extender) into vacuum. In the extender, the plasma generated by the arc cools down to about room temperature, and tungsten clusters grow. $\mathrm{W}_{n} \mathrm{~N}_{m}$ clusters can be generated by two different ways: A pulse of $\mathrm{N}_{2}$ gas is injected 2-3 cm downstream into the He flow and $\mathrm{N}_{2}$ molecules react with the clusters. Alternatively, a pulse of $\mathrm{N}_{2}$ gas is inserted into the source before the He valve opens to allow $\mathrm{N}_{2}$ being present in the electric arc, leading to the dissociation of a large fraction of the $\mathrm{N}_{2}$ molecules into atomic nitrogen. The mass of the clusters can be selected using a reflectron time-of-flight spectrometer. A 


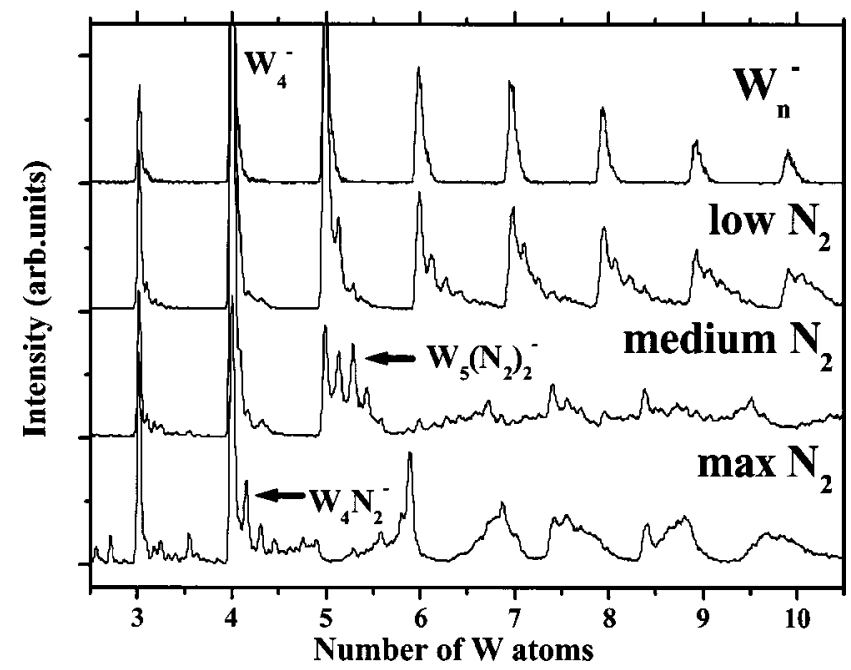

FIG. 1. Mass spectra of tungsten anion clusters before and after reactions in different background pressures of molecular $\mathrm{N}_{2}$.

mass-selected bunch is irradiated by a UV laser pulse (photon energy $=4.66 \mathrm{eV}$ ), and the kinetic energies of the detached electrons are measured using a "magnetic bottle"type time-of-flight electron spectrometer. The peaks in the photoelectron spectra correspond to vertical transitions from the anionic ground state to the allowed states of the neutral having the same geometry as the anion. The peak at the lowest binding energy corresponds to the transition into the neutral ground state and its position is the vertical position in the vertical detachment energy. Vibrational fine structures of this feature can be assigned to vibrational modes of the neutral species in the geometry of the anion.

\section{RESULTS AND DISCUSSIONS}

\section{A. Mass spectra of $\mathrm{W}_{n}^{-}$reacted in molecular and atomic nitrogen environments}

Mass spectra for the tungsten anion clusters taken before and after reaction with molecular nitrogen $\left(\mathrm{N}_{2}\right)$ are illustrated in Fig. 1. As the background pressure of $\mathrm{N}_{2}$ increases, concentrations of some pure tungsten clusters decrease, and concomitantly new peaks for $\mathrm{W}_{n} \mathrm{~N}_{m}^{-}$appear. $\mathrm{W}_{3}^{-}$do not react with nitrogen at all, whereas $\mathrm{W}_{4}^{-}$can be partly converted to $\mathrm{W}_{4} \mathrm{~N}_{2}^{-}$only at very high $\mathrm{N}_{2}$ background pressure, indicative of very weak $\mathrm{W}_{n}^{-}-\mathrm{N}_{2}$ interactions for $n=2-4$. With further increasing cluster size, the reactivity toward nitrogen adsorption becomes higher. It is important to note that the formation of $\mathrm{W}_{n} \mathrm{~N}_{m}^{-}$with $m=$ odd numbers is not observed, suggesting that the concentration of atomic nitrogen in the cluster source was negligibly low in this experiment.

Figure 2 shows the mass spectra of tungsten anion clusters taken before and after reaction in atomic nitrogen $(\mathrm{N})$ environments. Here, $\mathrm{W}_{n} \mathrm{~N}_{m}^{-}$with $m=$ odd numbers as well as even numbers are observed, indicating that tungsten clusters actually reacted with atomic nitrogen. For the low $N$ pressures, the $\mathrm{W}_{n} \mathrm{~N}^{-}$peak intensities are higher than those of the respective $\mathrm{W}_{n} \mathrm{~N}_{2}^{-}$peaks, indicating that the majority of tungsten clusters actually reacted with atomic nitrogen. In con-

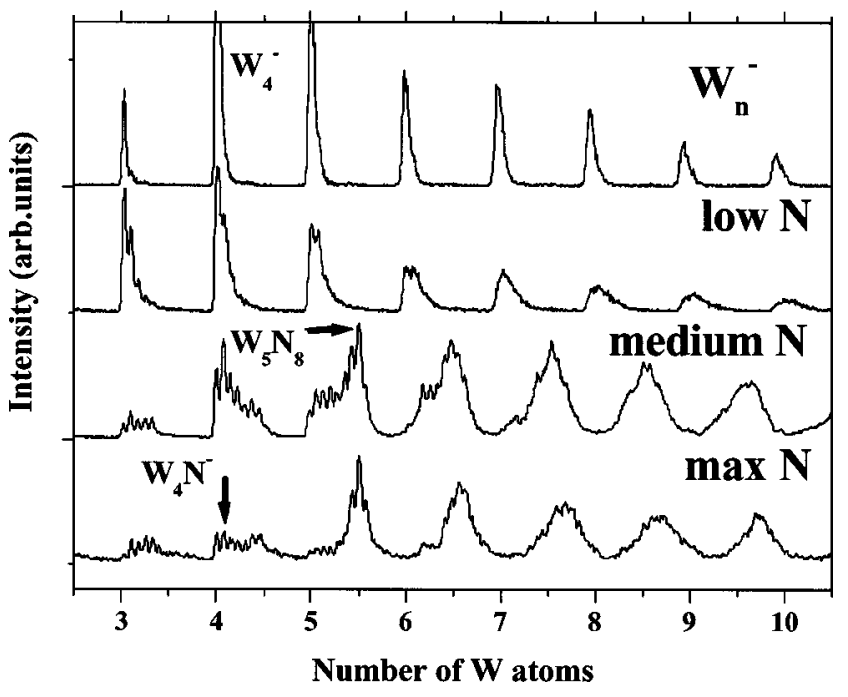

FIG. 2. Mass spectra of tungsten anion clusters before and after reactions with different atomic nitrogen background pressure.

trast to the case of molecular nitrogen, smaller clusters as well as larger clusters can readily react with atomic nitrogen to yield $\mathrm{W}_{n} \mathrm{~N}_{m}^{-}$clusters.

\section{B. UPS spectrum for $\mathrm{WN}_{2}^{-}$}

As a first example, the photoelectron spectrum of the reacted monomer $\mathrm{W}\left(\mathrm{N}_{2}\right)^{-}$is shown in Fig. 3. The spectrum is independent of the method of synthesis, i.e., the same spectrum is obtained by using molecular or atomic nitrogen. A broad feature with vibrational fine structures can be observed between 0.5 and $2.5 \mathrm{eV}$ binding energy. A spacing of $290 \pm 20 \mathrm{meV}$ between the main peaks $A$ and $B$ is slightly lower than the stretching frequency of free $\mathrm{N}_{2}$. We assign feature $A$ to the $0 \leftarrow 0$ and feature $B$ to the $1 \leftarrow 0$ vibrational transitions of $\mathrm{N}-\mathrm{N}$ stretching mode, implying that $\mathrm{N}_{2}$ does not dissociate. Since the intensity of the $1 \leftarrow 0$ transition is higher than the one of the $0 \leftarrow 0$ transition, further transitions into higher vibrational states of the neutral are expected corresponding to the "normal" Franck-Condon profile. However, higher vibrational state is not detected in Fig. 3, most likely due to the fact that the higher vibrationally excited states are not bound and undergo vibrational predissociation, which is very fast, resulting in the observation of broad vibrational transitions without discrete peaks.

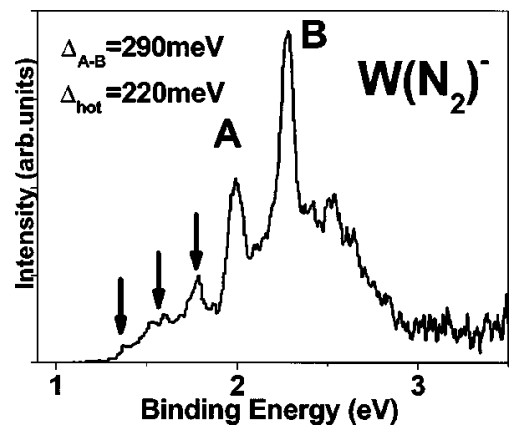

FIG. 3. UPS spectra of $\mathrm{W}_{1} \mathrm{~N}_{2}^{-}$. The same spectrum can be obtained using atomic and molecular nitrogen. 
Some weak "hot bands" are observed in Fig. 3 (marked by arrows at binding energies below $2 \mathrm{eV}$ ), which we assign to transitions from excited vibrational levels of the anion. This frequency is significantly lower than that of the neutral $\mathrm{WN}_{2}$, indicating that the $\mathrm{N}-\mathrm{N}$ bonding is more activated in the anionic state with respect to the neutral one. In contrast to the bulk W surface, chemisorption of vibrationally excited $\mathrm{N}_{2}$ to a $\mathrm{W}$ atom does not allow quenching of the vibrational excitation. The vibrational quantum of $\mathrm{N}_{2}$ is so large that collisions with $\mathrm{He}$ atoms in the extender cannot efficiently cool down the clusters. This explains our observation for the highly excited anions, although the vibrational temperature of anionic clusters created in our cluster source is usually estimated to be room temperature.

According to the qualitative analysis of the photoelectron spectra of $\mathrm{W}\left(\mathrm{N}_{2}\right)^{-}$described earlier, nitrogen is molecularly bound to the $\mathrm{W}$ anion. Because of the pronounced vibrational progression observed, the additional electron most likely occupies an orbital with a strong contribution of the antibinding $2 \pi^{*}$ orbital of $\mathrm{N}_{2}$. Accordingly, the additional electron of the anionic state further weakens the $\mathrm{N}-\mathrm{N}$ bond. From these considerations, it is reasonable to conclude that the propensity of the anions toward dissociative chemisorption should be stronger than that of the neutral counterpart, implying that a molecularly bound $\mathrm{N}_{2}$ is the most stable configuration not only for the anionic $\mathrm{W}\left(\mathrm{N}_{2}\right)^{-}$, but also for the respective neutral species.

\section{UPS spectra for $\mathrm{W}_{n} \mathrm{~N}_{2}^{-}(n=6-8)$}

For the larger $\mathrm{W}_{n} \mathrm{~N}_{2}^{-}$clusters with $n=6-8$ prepared using molecular nitrogen, UPS spectra show vibrational fine structures ${ }^{21}$ corresponding to about $200 \mathrm{meV}$ (Fig. 4). This value is attributed to the stretching frequency of chemisorbed $\mathrm{N}_{2}$ molecules based on previous high resolution electron energy loss spectroscopy data for $\mathrm{N}_{2}$ adsorption at liquid nitrogen temperature on tungsten single crystal surfaces, ${ }^{18}$ indicating that nitrogen is molecularly adsorbed on these clusters. These results are different from those from W(100), on which dissociative chemisorption of nitrogen takes place at room temperature. Our results can be interpreted in two different ways: Analogous to the case of tungsten bulk crystal surfaces, dissociative chemisorption of nitrogen on these tungsten clusters is thermodynamically more favorable than molecular adsorption, ${ }^{18,19}$ but the activation barriers for the dissociative adsorption are too high to be overcome at room temperature, ending up with molecular chemisorption. An alternative interpretation is that the formation of molecular nitrogen results from a larger thermodynamic stability of molecular chemisorption than dissociative one, i.e., on these tungsten clusters, the stable and metastable states of nitrogen adsorption are reversed with respect to bulk crystals.

In order to better understand the energetics of $\mathrm{N}_{2}$ adsorption on $\mathrm{W}_{n}^{-}$clusters with $n=6-8$, UPS spectra are collected for the $\mathrm{W}_{n} \mathrm{~N}_{2}^{-}$clusters prepared using atomic nitrogen environments (Fig. 4: $+2 \mathrm{~N}$ ). Considering that minor differences in UPS can come from different cluster temperatures, the positions and intensities of the features in the UPS spectra of $\mathrm{W}_{n} \mathrm{~N}_{2}^{-}(n=6,7)$ created by atomic and molecular nitrogen

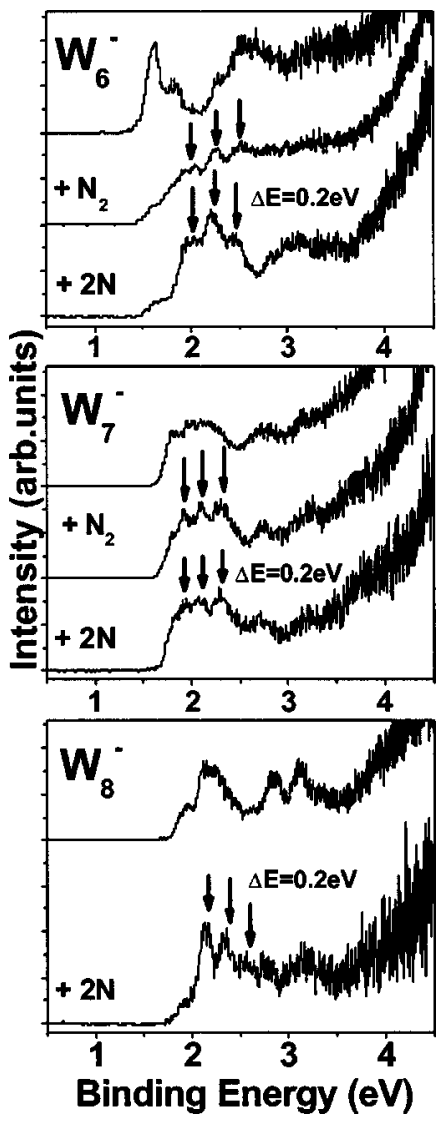

FIG. 4. UPS spectra of various $\mathrm{W}_{n}^{-}$and $\mathrm{W}_{n} \mathrm{~N}_{2}^{-}$clusters $(n=6-8) . \mathrm{W}_{n} \mathrm{~N}_{2}^{-}$ clusters were created by exposing tungsten clusters to atomic $(+2 \mathrm{~N})$ or molecular $\left(+\mathrm{N}_{2}\right)$ nitrogen. The arrows mark peaks with a spacing of 200 $\mathrm{meV}$, which are tentatively assigned to the stretching frequency of molecularly adsorbed $\mathrm{N}_{2}$ (see the text). In case of $\mathrm{W}_{8}^{-}$reacted with molecular $\mathrm{N}_{2}$ the cluster intensity was too low for recording a spectrum.

are almost identical. UPS spectra for these clusters also show fine structures corresponding to about $200 \mathrm{meV}$, indicative of the formation of di-nitrogen species, i.e., two nitrogen atoms adsorbed on these clusters can spontaneously recombine to form di-nitrogen species. Molecular adsorption of $\mathrm{N}_{2}$ is energetically more stable than dissociative chemisorption, and the mobilities of nitrogen atoms on these clusters are quite high. The energy diagram relevant for the nitrogen adsorption on these clusters is illustrated in Fig. 5. This energy diagram illustrates that the molecularly bound $\mathrm{N}_{2}$ is energetically more stable than the dissociatively bound one, which is reversed with respect to the energetics on tungsten bulk crystals.

We cannot rule out the possibility that tungsten clusters did not react with atomic nitrogen but selectively with small amounts of molecular nitrogen, existing in atomic nitrogen environments. If dissociative chemisorption was energetically more favorable than the molecular one, the tungsten cluster would react better with atomic nitrogen than molecular nitrogen. That the tungsten clusters preferentially react with molecular nitrogen $\left(\mathrm{N}_{2}\right)$ indicates that molecular chemisorption of nitrogen is energetically more stable than dissociative chemisorption. Thus, the energy diagram in Fig. 5 is still valid within this scenario. These results are much different from those found for tungsten bulk crystals, on 


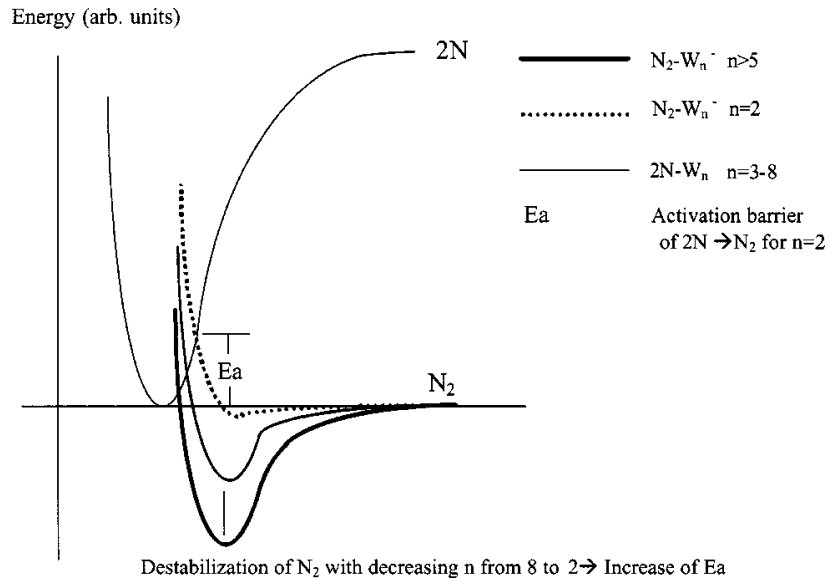

FIG. 5. Potential energy diagram for the nitrogen chemisorption on tungsten anion clusters consisting of eight atoms or less.

which dissociation of nitrogen is thermodynamically more favored with respect to the molecular chemisorption. ${ }^{18,19}$

Our experimental observations that molecular nitrogen is more stable than atomic nitrogen on $\mathrm{W}_{n}^{-}$with $n=6-8$ can be rationalized by an electronic model, in which metal to adsorbate charge transfer is important for the dissociative adsorption. The required energy to remove electrons from tungsten is decreased with increasing cluster size, since larger clusters with higher average coordination numbers can more effectively screen the residual positive hole created by removal of an electron. ${ }^{16,22}$ This kind of final state screening effects is evident in core level and valence level shift experiments, in which binding energies of electrons shift to higher energies with decreasing cluster size. ${ }^{23}$ Similarly, the less effective screening of the positive holes in smaller clusters reduces the charge transfer from tungsten to $\mathrm{N}_{2}$. Insufficient charge transfers to the $\mathrm{N}_{2} 2 \pi^{*}$ orbital cannot separate two nitrogen atoms, lowering the energy gain of the dissociative chemisorption in comparison with those of the molecular adsorption. It is worth mentioning that the variation of the CO dissociation probability on different $3 d$ metals strongly correlates with the work function change, in line with our suggestion that the effective (i.e., including final state effects) electron binding energy-in our case of the W $5 d$-orbitals-in the substrate is an important factor to determine the thermodynamic stability of the dissociative chemisorption of diatomic molecules. ${ }^{24}$

Similar to the arguments used for $\mathrm{WN}_{2}^{-}$, the additional electron in the anionic state should occupy an orbital with a strong contribution of the antibinding $2 \pi^{*}$ orbital of $\mathrm{N}_{2}$, in other words the additional electron of the anion further weakens the $\mathrm{N}-\mathrm{N}$ bond. Taking this into account, we suggest that the propensity of the anions toward dissociative chemisorption should be stronger than that of the neutral counterpart, implying that a molecularly bound $\mathrm{N}_{2}$ is the most stable configuration not only for the anionic $\mathrm{W}_{n}\left(\mathrm{~N}_{2}\right)^{-}$with $n$ $=6-8$ but also for the respective neutral species.

It is a generally accepted view that the activation barrier of dissociative chemisorption is sensitive to the cluster size, ${ }^{2}$ whereas less attention has been paid to the variation of thermodynamic parameters of chemisorption as a function of cluster size. Our results demonstrate that the thermodynamic stabilities of dissociative and molecular adsorption are strongly dependent on cluster size.

This size-dependent variation of chemisorption energetics can be rationalized within an electronic model. However, it is obvious that a complete understanding on the adsorptive behaviors of nitrogen molecules on metal clusters can only be obtained by considering geometrical shapes of clusters as well as their electronic structures, implying that further theoretical studies on this issue are required. Our experimental data provide an important base line for future studies.

\section{UPS spectra for $\mathrm{W}_{n} \mathrm{~N}_{2}^{-}(n=2-5)$}

In contrast to the results for $n=6-8$, UPS spectra for $\mathrm{W}_{n} \mathrm{~N}_{2}^{-}$with $n=2-5$ depend strongly on the source of nitrogen, i.e., atomic nitrogen and molecular nitrogen yield different UPS results (Fig. 6). $\mathrm{W}_{2}^{-}$and $\mathrm{W}_{3}^{-}$did not react at all with molecular nitrogen at room temperature even at the highest $\mathrm{N}_{2}$ background pressure in our experiments, thus no UPS spectrum is available for $\mathrm{W}_{2}^{-}+\mathrm{N}_{2}$ and $\mathrm{W}_{3}^{-}+\mathrm{N}_{2}$. For $\mathrm{W}_{n}^{-}$with $n=4,5$ almost no modification of the electronic structures for the tungsten clusters can be observed upon reaction with $\mathrm{N}_{2}$ to form $\mathrm{W}_{n} \mathrm{~N}_{2}^{-}$, indicating very weak van der Waals interactions between $\mathrm{N}_{2}$ and these tungsten clusters.

When atomic nitrogen is used instead of molecular nitrogen to produce $\mathrm{W}_{n} \mathrm{~N}_{2}^{-}$with $n=2-5$, the UPS spectra are significantly modified with respect to those of the respective pure $\mathrm{W}_{n}^{-}$clusters, indicative of quite strong nitrogentungsten interactions (Fig. 6). Our results for $\mathrm{W}_{2} \mathrm{~N}_{2}^{-}$and $\mathrm{W}_{3} \mathrm{~N}_{2}^{-}$are suggestive of atomic chemisorption, since molecular adsorption of nitrogen on $\mathrm{W}_{2}^{-}$and $\mathrm{W}_{3}^{-}$was shown to be unstable. For $\mathrm{W}_{4}^{-}$, the vibrational fine structure of the most intense feature at $2.1 \mathrm{eV}$ binding energy corresponds to $60 \mathrm{meV}$, which can be attributed to the $\mathrm{W}-\mathrm{N}$ stretching mode, ${ }^{18}$ suggesting that nitrogen is atomically bound in this case. For $\mathrm{W}_{5} \mathrm{~N}_{2}^{-}$synthesized using atomic nitrogen, vibrational fine structures can be hardly identified in the UPS spectrum, however the UPS spectrum of this cluster consisting of three distinct peak is almost identical to those of $\mathrm{W}_{4} \mathrm{~N}_{2}^{-}$, suggesting that nitrogen atoms adsorbed on both clusters exhibit very similar structures. Thus, we propose that analogous to $\mathrm{W}_{4}^{-}, \mathrm{W}_{5}^{-}$do not allow recombination of two nitrogen atoms to di-nitrogen species.

Considering the cluster-size-dependent changes of the hole screening effect aforementioned, the charge transfers between $\mathrm{N}_{2}$ and $\mathrm{W}_{n}^{-}$for $n=2-5$ are expected to be weaker than those for $n>5$. TOF mass spectroscopy experiments in Fig. 1 reveal that reactivities of $\mathrm{W}_{n}^{-}$(for $n=3-8$ ) toward $\mathrm{N}_{2}$ adsorption become lower with decreasing cluster size, which seems to reconcile with the weaker charge transfers for the smaller clusters. In this context, the experimental results in Fig. 6 that nitrogen atoms on $\mathrm{W}_{n}^{-}$with $n=3,4,5$ do not recombine to $\mathrm{N}_{2}$ are surprising, since the stabilization of atomically chemisorbed nitrogen can be regarded as indicative of a larger charge transfer.

Our observation for the atomically chemisorbed nitrogen in $\mathrm{W}_{n} \mathrm{~N}_{2}^{-}(n=2-5)$ can be rationalized taking into account 


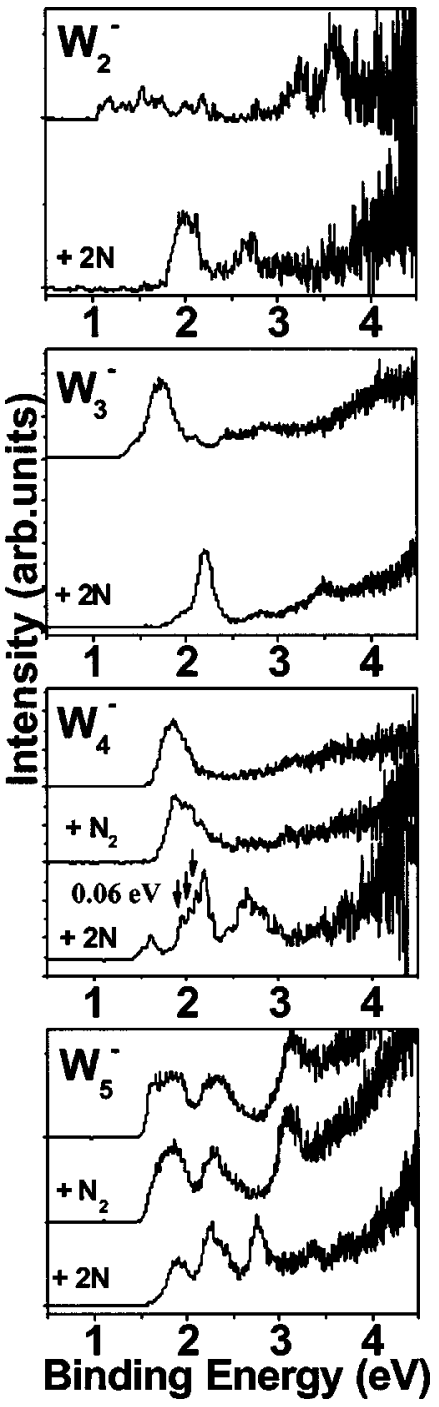

FIG. 6. UPS spectra of various $\mathrm{W}_{n}^{-}$and $\mathrm{W}_{n} \mathrm{~N}_{2}^{-}$clusters $(n=2-5) . \mathrm{W}_{n} \mathrm{~N}_{2}^{-}$ clusters were created by exposing tungsten clusters to atomic $(+2 \mathrm{~N})$ or molecular $\left(+\mathrm{N}_{2}\right)$ nitrogen. In case of $\mathrm{W}_{2}^{-}$and $\mathrm{W}_{3}^{-}$reacted with molecular $\mathrm{N}_{2}$ no cluster signal was found.

that more pronounced upwards shifts of the $\mathrm{W}-\mathrm{N}_{2}$ potential curve with respect to those of the $\mathrm{W}-2 \mathrm{~N}$ curve increase the activation barrier for the transition from the metastable state (atomic chemisorption) to the ground state (molecular chemisorption) (Fig. 5). Majority of nitrogen atoms in the gas phase interacting with tungsten clusters may fall into the potential well of $\mathrm{W}_{n}^{-}-\mathrm{N}_{2} \quad(n=2-5)$, be temporarily trapped in the ground state, and then decomposed to $\mathrm{N}_{2}$ and $\mathrm{W}_{n}^{-}$due to the very low $\mathrm{N}_{2}-\mathrm{W}_{n}^{-}$bonding energies. In contrast, some nitrogen atoms moving along the potential curve of $\mathrm{W}_{n}^{-}-2 \mathrm{~N}$ cannot easily escape the potential well because of the increased activation barrier between the metastable and ground states, thus they are quenched in the metastable state.

\section{CONCLUSION AND OUTLOOK}

UPS and TOF mass spectrometers were used to study structures of $\mathrm{W}_{n} \mathrm{~N}_{2}^{-}$clusters produced by exposing tungsten clusters to molecular and atomic nitrogen environments. For
$\mathrm{W}_{1} \mathrm{~N}_{2}^{-}$, molecular adsorption of nitrogen is observed. For the tungsten anion clusters smaller than $\mathrm{W}_{9}^{-}$, molecular adsorption of nitrogen was found to be thermodynamically more stable with respect to the dissociative chemisorption. In contrast to our results, dissociative adsorption of nitrogen is energetically more favorable than molecular adsorption on tungsten single crystal surfaces, ${ }^{18,19}$ suggesting that the ground state of nitrogen chemisorption undergoes transition from molecular to dissociative adsorption $\left(\mathrm{N}_{2} \longrightarrow 2 \mathrm{~N}\right)$ with increasing number of tungsten atoms $(n)$. It is important to note that sharp increase in the reactivity of $\mathrm{W}_{n}$ toward nitrogen adsorption was observed at $n=15$ in Refs. 6 and 7, which may be indicative of the $\mathrm{N}_{2} \rightarrow 2 \mathrm{~N}$ transition at this cluster size. It is likely that similar variations of the chemisorption energetics upon decreasing cluster size can occur for other metal/gas systems.

Comparative studies for adsorption of atomic and molecular species of diatomic molecules on metal clusters can be expected to play an important role for unveiling molecular-level mechanisms of various catalytic reactions on nanomaterials. For example, we have recently provided evidence for the stabilization of di-oxygen species on $\mathrm{Au}_{2}^{-}$and $\mathrm{Au}_{4}^{-}$clusters, which are suggested to play a pivotal role for $\mathrm{CO}$ oxidation on gold catalysts. ${ }^{25}$ Yet, it is not clear if our observation for the molecular oxygen species on gold clusters is due to larger thermodynamic stabilities of molecular oxygen with respect to atomic oxygen, or increased activation barrier for the dissociation of oxygen, which is energetically more stable than molecular adsorption. Comparative studies for the adsorption of atomic and molecular oxygen on gold anion clusters are on the way to provide an answer to this question.

\section{ACKNOWLEDGMENT}

Financial support from Deutche Forschungsgemeinschaft (DFG) is gratefully acknowledged.

${ }^{1}$ A. Sanchez, A. Abbet, U. Heiz, W.-D. Schneider, H. Häkkinen, R. N. Barnett, and U. Landman, J. Phys. Chem. A 103, 9573 (1999).

${ }^{2}$ M. Valden, X. Lai, and D. W. Goodman, Science 281, 1647 (1998).

${ }^{3}$ A. Kaldor, D. M. Cox, and M. R. Zakin, Adv. Chem. Phys. 70, 211 (1988).

${ }^{4}$ M. B. Knickelbein, Annu. Rev. Phys. Chem. 50, 79 (1999).

${ }^{5}$ W. T. Wallace and R. L. Whetten, J. Am. Chem. Soc. 124, 7499 (2002).

${ }^{6}$ S. A. Mitchell, D. M. Rayner, T. Bartlett, and P. A. Hackett, J. Chem. Phys. 104, 4012 (1996).

${ }^{7}$ L. Holmgren, M. Andersson, and A. Rosen, J. Chem. Phys. 109, 3232 (1998).

${ }^{8}$ J. Conceicao, R. T. Laaksonen, L.-S. Wang, T. Guo, P. Nordlander, and R. E. Smalley, Phys. Rev. B 51, 4668 (1995).

${ }^{9}$ R. L. Whetten, D. M. Cox, D. J. Trevor, and A. Kaldor, Phys. Rev. Lett. 54, 1494 (1985)

${ }^{10}$ H. Kietzmann, J. Morenzin, P. S. Bechthold, G. Gantefoer, and W. Eberhardt, J. Chem. Phys. 109, 2275 (1998).

${ }^{11}$ J. L. Elkind, F. D. Weiss, J. M. Alford, R. T. Laaksonen, and R. E. Smalley, J. Chem. Phys. 88, 5215 (1998).

${ }^{12}$ M. D. Morse, M. E. Geusic, J. R. Heath, and R. E. Smalley, J. Chem. Phys. 83, 2293 (1985).

${ }^{13}$ M. R. Zakin, R. O. Brickman, D. M. Cox, and A. Kaldor, J. Chem. Phys. 88, 3555 (1988).

${ }^{14}$ R. L. Whetten, M. R. Zakin, D. M. Cox, D. J. Trevor, and A. Kaldor, J. Chem. Phys. 85, 1697 (1986).

${ }^{15}$ D. M. Cox, K. C. Reichmann, D. J. Trevor, and A. Kaldor, J. Chem. Phys. 88, 111 (1988). 
${ }^{16}$ S. Burkart, N. Blessing, and G. Ganteför, Phys. Rev. B 60, 15639 (1999).

${ }^{17}$ K. Jakobi, Phys. Status Solidi A 177, 37 (2000).

${ }^{18}$ A. Sellidj and J. L. Erskine, Surf. Sci. 220, 253 (1989).

${ }^{19}$ The Chemical Physics of Solid Surfaces and Heterogeneous Catalysis, edited by King and Woodruff (1990), Vol. 3A.

${ }^{20}$ S. Burkart, N. Blessing, B. Klipp, J. Müller, G. Ganteför, and G. Seifert, Chem. Phys. Lett. 301, 546 (1999).

${ }^{21}$ For $\mathrm{W}_{8}^{-}+\mathrm{N}_{2}$, a spectrum with sufficient signal to noise ratio to allow unambiguous assignment could not be recorded. Note that for larger clusters, separation of $\mathrm{W}_{n} \mathrm{~N}_{2}^{-}$from the bare $\mathrm{W}_{n}$ is very difficult due to broader isotope distributions.

${ }^{22}$ K.-H. Meiwes-Broer, Hyperfine Interact. 89, 263 (1994).

${ }^{23}$ T. P. St. Clair and D. W. Goodman, Top. Catal. 13, 5 (2000).

${ }^{24}$ R. Hoffman, Rev. Mod. Phys. 60, 601 (1988).

${ }^{25}$ D. Stolcic, M. Fischer, G. Ganteför, Y. D. Kim, Q. Sun, and P. Jena, J. Am. Chem. Soc. 125, 2848 (2003). 